\title{
NEW ESTIMATION TECHNIQUE FOR A CLASS OF CHAOTIC SIGNALS
}

\author{
Dinesh Rajan and Behnaam Aazhang \\ Department of Electrical and Computer Engineering, \\ Rice University, 6100 S.Main Street, \\ Houston, TX 77005. \\ dinesh@rice.edu, aaz@rice.edu
}

\begin{abstract}
We propose a new technique for the estimation of chaotic signals in the presence of additive noise. The new method uses statistical measures to restrict the space over which the signals are received. The proposed technique is applicable to a large variety of chaotic signals and has good performance indicated by the low estimation error bias and variance. The complexity of the algorithm is shown to be low.
\end{abstract}

\section{INTRODUCTION}

Chaotic systems have very desirable properties for signal analysis and synthesis. Although they produce random noise like signals, they are actually the output of deterministic systems. They also have a very wide frequency spectrum that make it difficult to intercept and detect. As a result they are very promising candidates for application in communication systems. The key feature of these systems is their sensitive dependence on initial conditions.

The important issue in using a chaotic signal for communication is the existence of a 'good' receiver and the ease of its implementation. Maximum likelihood estimation algorithms are known only for a very limited class of chaotic signals. Characteristics of asymptotic maximum likelihood estimators for chaotic signals are derived in [1] and a specific maximum likelihood estimator for the tent map is given in [4].

In this paper we propose a new technique for the estimation of a wide range of chaotic signals. The proposed algorithm is not restricted to AWGN channels but could also be applied to any kind of additive noise channels. We show that the new algorithm gives lower error variances than existing algorithms. The proposed algorithm is not restricted to piecewise linear or unimodal maps.

\section{SIGNAL AND CHANNEL MODELS}

We consider the estimation of signals that are corrupted by additive white noise. The channel model under consideration is given by

$$
y_{n}=x_{n}+z_{n},
$$

where $x_{n}$ is the transmitted signal, $y_{n}$ is the received signal, $z_{n}$ is the noise component and $n$ represents the time index. The transmitted signal sequence $x_{n}$ is generated as the output of a chaotic map. In this paper we restrict ourselves to one-dimensional maps as specified below.

$$
x_{n}=f\left(x_{n-1}\right)
$$

where $f($ ) is a non linear function. However, we do not restrict ourselves to unimodal or piecewise linear maps, which is a requirement on other algorithms like those proposed in [2]. Some examples of chaotic maps are given in Fig 1 . The additive noise is not assumed to have Gaussian distribution in the estimation algorithm.

\section{ESTIMATION TECHNIQUE}

Our basic problem is to find an estimate of the transmitted signal $x_{n}$ for $\mathrm{n}=1,2, \ldots \mathrm{N}$. This problem reduces to accurately estimating the initial 

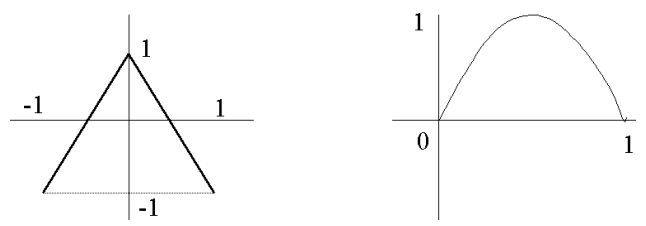

Figure 1: Examples of one dimensional chaotic maps : tent map and logistic map

transmitted signal ( or initial condition of the map ). However, using steepest gradient methods to directly estimate the intial condition is not possible because of the fractal nature of the estimation error [3].

Instead [4] suggests a scheme wherein the iternary of the signal is first determined and this information is used to find the initial condition. The itenary of a chaotic sequence $x_{1}, x_{2}, \ldots, x_{N}$ generated from a unimodal map is defined by

$$
\begin{aligned}
p_{n} & =0 \text { if } 0 \leq x_{n}<c \\
& =1 \text { if } c \leq x_{n}<1
\end{aligned}
$$

for $\mathrm{n}=1,2, \ldots, \mathrm{N}-1$ and $\mathrm{c}$ is the value of $\mathrm{x}$ for which $\mathrm{f}(\mathrm{x})$ is maximum. This definition can be easily modified to include other maps like those in Fig 1. We will illustrate our algorithm for the tent map which is given by

$$
f(x)=\beta-1-\beta(x),
$$

where $1<\beta \leq 2$.

Assume initially that we are operating in the high SNR region. The main idea is that if the itenary of the transmitted signals is known then we can effectively use a steepest descent algorithm to estimate the final condition. Using this estimate of the final condition and the itenary we can find an estimate of the initial condition. Our contention is that with a large probability the itenaries of the received signal and transmitted signal are almost equal. So we propose that we use a test to search over a subspace of possible itenaries.

For the case of tent map, the itenary of the signal sequence is the same as the sign of the transmitted signal sequence. We include all possible values for the $i^{\text {th }}$ component of the iternary if $\left|y_{i-1}-\alpha_{1}\right| \leq \epsilon$ or $\left|y_{i-1}-\alpha_{2}\right| \leq \epsilon$ where $\alpha_{1}$ and $\alpha_{2}$ are the zeros of $f()$. Otherwise we take the iternary to be equal to the sign of the received signal. Using this we form a list of most likely itenaries.

It can be easily shown that the probability of not including the correct itenary of the transmitted signal sequence in the list of possible itenaries can be approximated by

$$
2 \operatorname{Pr}\left(\alpha-\epsilon<x_{n}<\alpha+\epsilon\right) \operatorname{Pr}\left(z_{n}>\epsilon\right)
$$

which reduces to $2 \epsilon Q\left(\frac{\epsilon}{\sigma}\right)$ under the assumption that $\mathrm{z}$ is $\mathrm{N}\left(0, \sigma^{2}\right)$ and $x_{n}$ is uniformly distributed between -1 and 1 , which is an invariant density for the tent map.

The plot of this probability for different $\epsilon$ is given in Fig 2 for Gaussian noise case at an SNR of $27 \mathrm{~dB}$. It is clear from the figure that for any value of $\epsilon$ the probability that our subset of itenaries will not include the correct itenary is below $10^{-3}$. The size of the search set depends on the value of $\epsilon$ and can be used to get a tradeoff between complexity and performance.

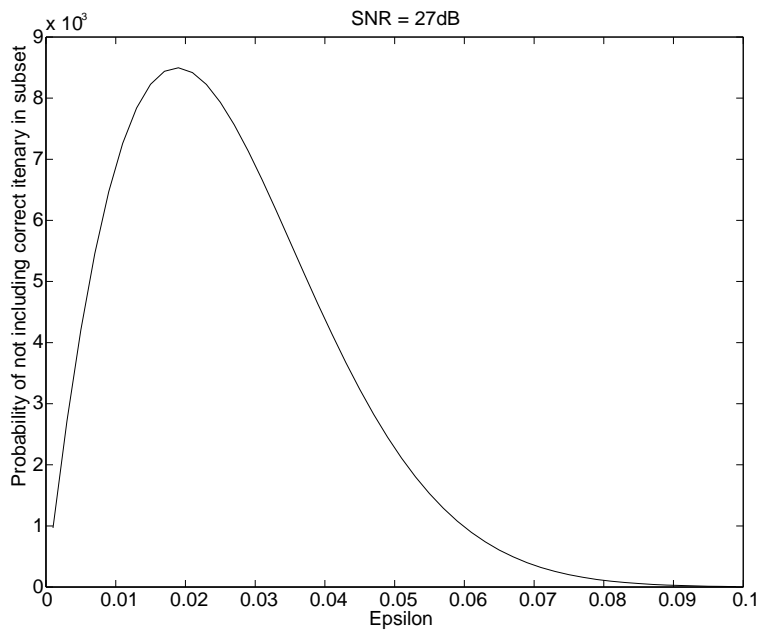

Figure 2: Error in not including the correct itenary in our subset of possible iternaries for tent map, SNR of $20 \mathrm{~dB}$

Once we have the set of possible itenaries, we perform a grid based search for the final value of the transmitted signal for each of these itenaries. We then jointly choose the itenary and final condition that gives the minimum probability of error. The complexity can be further lowered by using a non-uniformly spaced grid to search for the final value of the transmitted sequence. Since we are 
only searching over a subset of possible iternaries, we call our new estimator as subspace based estimator.

The new subspace algorithm is summarized below

- Choose an $\epsilon$ based on knowledge of SNR and amount of computation allowed

- Form the subset of possible iternaries based on whether or not $\left|y_{i-1}-\alpha_{1}\right| \leq \epsilon$ or $\mid y_{i-1}-$ $\alpha_{2} \mid \leq \epsilon$ where $\alpha_{1}$ and $\alpha_{2}$ are the zeros of $\mathrm{f}()$.

- For each itenary in selected subset estimate the final value $x_{N}$ using a grid search.

- Choose the itenary and final value that gives the lowest estimation error

\section{RESULTS}

The plot of the estimation error versus SNR is shown in Fig 4 for the tent map and in Fig 3 for the logistic map for a AWGN channel. From this figure it is clear that our error is lower than the Cramer Rao bound and consequently biased. For the logistic map we have indicated the performance of the subspace estimator for two different values of resolution of grid for estimating the final value. The subspace of possible iternaries over which we search is maintained the same in both the cases. As can be expected the higher resolution search achieves Cramer-Rao bound whereas the lower resolution search performs badly especially at high SNR's. The plot of the estimator bias is shown in Fig 5. It is clear from the figure that the estimator bias is quite small especially for high SNR's. It is our conjecture that at high SNR's the estimator is essentially unbiased and achieves the Cramer-Rao bound.

\section{CONCLUSIONS}

We have introduced a new technique for the estimation of a wide range of chaotic signals. The new technique involves enumeration of a set of most likely itenaries and then jointly estimating the final condition and itenary of the map. This subspace based search algorithm has low implementa-

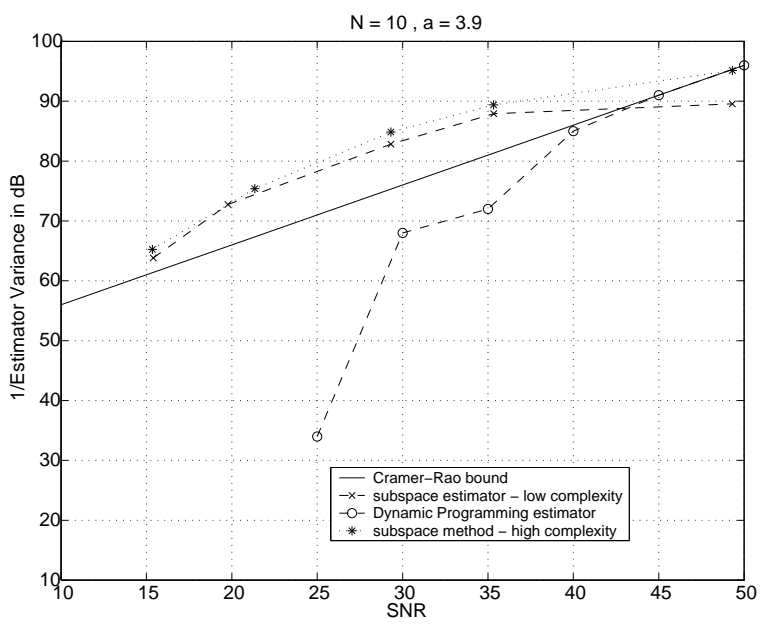

Figure 3: Estimator Variance for logistic map

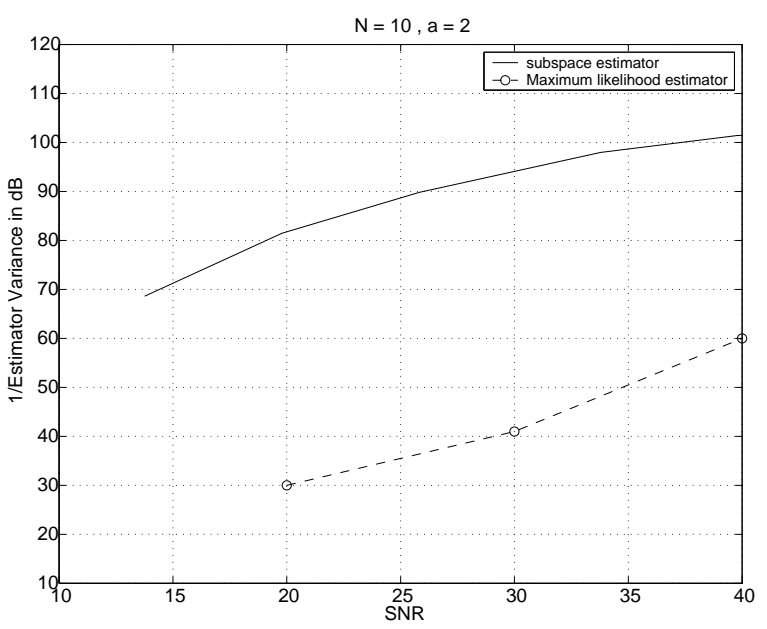

Figure 4: Estimator Variance for tent map

tion complexity and low estimator error variance. It is applicable to all kinds of additive noise channels and not necessarily Gaussian channels. It can also be easily extended to the case in which some known function of the chaotic signal is observed in noise. This will encompass the case of slowly fading or block fading channels. In the case of multiple chaotic signals using the same physical medium and interfering with one another different methods will be needed to achieve single user performance. 


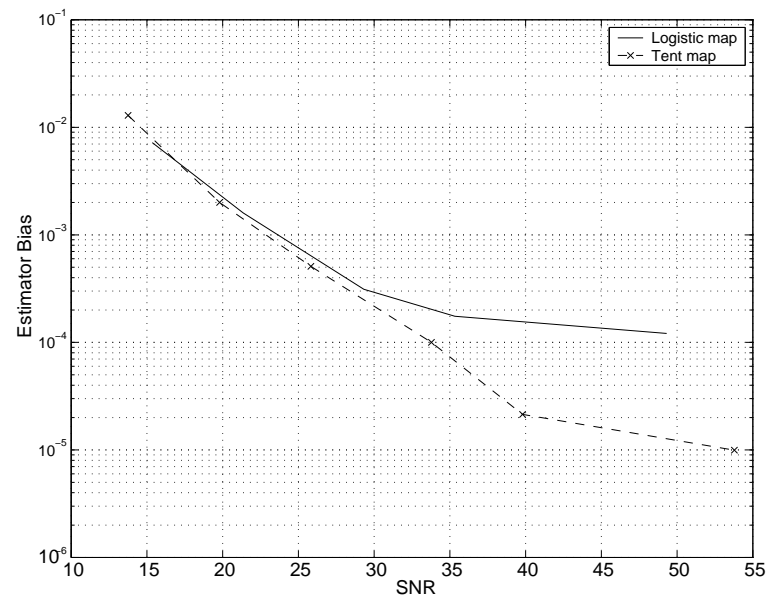

Figure 5: Estimator bias for tent and logistic map

\section{REFERENCES}

[1] Steven Kay. Asymptotic maximum likelihood estimator performance for chaotic signals in noise. IEEE Transactions on Signal Processing, 43:1009-1012, 1995.

[2] Steven Kay and Venkatesh Nagesha. Methods for chaotic signal estimation. IEEE Transactions on Signal Processing, 43:2013-2016, 1995.

[3] Cory Myers, Steven Kay, and Michael Richard. Signal separation for nonlinear dynamical systems. Proceedings of ICASSP, 4:129-132, 1992.

[4] Haralabos C. Papadopoulos and Gregory Wornell. Maximum-likelihood estimation of a class of chaotic signals. IEEE Transactions on Information Theory, 41:312-317, 1995. 